\title{
Evolución conceptual y utilidad social de la Geografía como materia de enseñanza
}

\author{
José R. Diaz Alvarez *
}

\section{REFLEXIONES SOBRE LAS DIFICULTADES EPISTEMOLOGICAS DE LA GEOGRAFIA}

Con demasiada insistencia se viene haciendo referencia a las nuevas corrientes, conceptuales o metodológicas, que pretenden dar a la Geografía un peso suficiente y un valor indiscutible en el marco general de la Ciencia y en el particular de las Ciencias Sociales. Tal esfuerzo en la investigación de fórmulas más universales para nutrir y definir el saber geográfico es una consecuencia de la falta de personalidad científica de la propia Geografía. Entre los geógrafos existe como un cierto miedo a la descalificación social. Hay un oculto sentimiento de culpabilidad, propio del intrusismo, por estar ejerciendo como científico en una disciplina que no termina de abrirse hueco entre los "saberes cientificos" por antonomasia (Fisica, Quimica, Matemáticas, entre las ciencias clásicas, o Genética, Informática, Robótica, entre las modernas). Por ello, el geógrafo necesita ejercitarse en su propia identidad, y esta autoafirmación sólo puede hacerla desde su propia materia de estudio.

De este modo, las denominadas grandes «revoluciones conceptuales" que la Geografía ha padecido durante los últimos 30 años (tendencias cuantitativa y radical, enfoques fenomenológico y perceptivo, influencias economicista y ecologista, etc.) no son otra cosa que forcejeos en la búsqueda de la ubicación definitiva en el dominio de las ciencias con prestigio social. El

( $\left.{ }^{\star}\right)$ Dr. en Geografía. Catedrático de Bachillerato INEM. Alhadra (Almería) 
construir una ciencia a partir de una parcela del conocimiento es una cuestión de método, de la utilización de un adecuado método: todo saber que pueda ser catalogado o tipificado que siga unas pautas de comportamiento suceptibles de ser regladas, que pueda predecir resultados en función de su propia dinámica y que pueda ir construyéndose a sí mismo a partir de sus propias premisas, es un saber científico. La Geografía puede ser ciencia con tal de que utilice sólo aquellos aspectos que permitan los desarrollos que hemos señalado. Ello no es difícil. Sin embargo, las ciencias tienen su justificación en la aceptación generalizada, al margen de sus propios cultivadores; cuando esa aceptación se produce, la ciencia está bautizada y en condiciones de emprender el recorrido de su propia maduración.

Admitiendo el símil biológico, toda ciencia pasa por una etapa infantil, periodo descriptivo de la ciencia, en el que se utiliza fundamentalmente a la observación como método de trabajo; una etapa juvenil, periodo de afirmación explicativa, basado en la sistematización y en la elaboración de una estructura científica; una etapa de madurez que se corresponde con la etapa tecnológica de las ciencias y se identifica con el período de reconocimiento social de un saber en función de su utilidad inmediata. La Geografía no ha sido ajena a esta evolución, aunque podríamos decir que ha tardado bastante en madurar y que su recorrido ha sido demasiado penoso, en parte por la amplitud de su campo de acción, que en la medida en que se iba definiendo se iba independizando con cuerpo doctrinal propio (Geología, Climatologia, Edafologia, Ecología, Demografía, etc.), en parte por la heterogeneidad de sus métodos, que deben responder a la doble exigencia de ser una ciencia de la naturaleza y una ciencia del hombre. Cada etapa del desarrollo de las ciencias geográficas ha atravesado sus propias crisis, que, si bien le han permitido un avance más consistente, han servido de base para el confusionismo epistemológico de esta ciencia y para la proliferación de tendencias que no son otra cosa que alternativas posibles a la salida de la correspondiente crisis.

En la valoración de la evolución de la Geografía hacia su madurez científica es posible que no se haya considerado siempre el estadio en que se encontraba el saber geográfico, con lo que se ha podido cometer el error de considerar avance lo que sólo era una variación formal y no esencial. Los auténticos pasos en esa evolución son los que han permitido la superación de fases, es decir, los que le han posibilitado el camino desde su estadio descriptivo al explicativo, y luego los que han facilitado la entrada del co- 
nocimiento geográfico en la etapa tecnológica. Esas geografias del progreso han sido calificadas, con evidente fortuna, como «Nuevas Geografías»" .

\section{El modelo descriptivo}

Desde Estrabón hasta Reclús, durante diecinueve siglos, la Geografia recorre un largo camino descriptivo; demasiado tiempo para una ciencia en plenitud de salud. La falta de desarrollo hay que buscarla en la adecuación de su oferta con las exigencias de la demanda social: el conocimiento de la toponimia de los pueblos a partir de largas relaciones de nombres ligados a la descripción de lugares, la relación ordenada de listas de nombres y detalles geográficos que constituyen el campo y contenidos de la Corografia, la narración fantástica o exagerada de experiencias exploratorias, eran suficientes para satisfacer una curiosidad más morbosa que utilitarista y una necesidad de erudición más personal que social. En sentido estricto no puede decirse que el modelo descriptivo abandone todo intento explicativo, pero las explicaciones sólo alcanzaban a los particularismos, por lo que la base didáctica de los mismos era más casual que causal. El molde de la descripción permite la referencia al detalle relacionado, pero no se detiene en la causa; pretende ser útil, pero no se plantea la posibilidad de la interacción con el propio espacio que describe para hacer un mejor uso del mismo, sino que su utilidad queda intrínseca en el propio acto del conocer. La Geografía Descriptiva cultivada durante la Edad Antigua y la Edad Media cabalgaba entre el relato fantástico y lo maravilloso, no habiendo escapado todavía al contenido mítico que la envolvía desde la época griega. En la Edad Moderna, a pesar del enorme éxito de algunas Cosmografías ${ }^{2}$, la finalidad explicativa todavía quedaba ausente en los tratados, con lo que el vigor y la estructura de la ciencia permanecia al margen de lo geográfico. El "siglo de las luces" que sentó las bases baconianas de la experimentación instituyendo el empirismo científico, que tan buenos resultados iba a proporcionar al progreso de los siglos siguientes, sólo rozó ligeramente en la elaboración del pensamiento geográfico e influyó en algunos trabajos como los de

' No es sólo el caso de las tendencias recientes que fueron calificadas a partir de la década de los 50 del presente siglo como "Nueva Geografia", sino que ya el gran Eliseo Reclús utilizó tal apelativo advirtiendo que era autor de un nuevo concepto de lo geográfico.

${ }^{2}$ En especial la del germano Sebastián MÜNSTER (1489-1552) cuya Cosmografia alcanzó una difusión universal a través de sus 55 ediciones en nueve idiomas. 
Buache ${ }^{3}$ (apenas conocido para los geógrafos) o los de Von Thünen que tanta difusión ha tenido entre los cultivadores de la sistemática en nuestro siglo. Humboldt y Ritter, tantas veces citados como los padres de la Geografía moderna, acabarian de dar el salto entre las etapas infantil y juvenil de nuestra ciencia.

\section{El proyecto explicativo}

El auténtico paso hacia "la juventud» de las ciencias geográficas se da cuando, metodológicamente, la Geografía se hace explicativa, tratando de investigar el porqué de los fenómenos geográficos. El camino hacia esta nueva etapa se inicia con la Geographia Generalis del alemán Bernhard VARENIO (1622-1650) que trata de ordenar el ámbito de los fenómenos, de los procesos y de los resultados de los componentes que intervienen de manera convergente en las acciones desarrolladas sobre la superficie de la Tierra y en estrecha y directa relación con ella. La fecundidad de la etapa juvenil lleva a la Geografía a su constitución como auténtica ciencia, a través de diversos balbuceos que unas veces consideran la originalidad de lo geográfico como elemento sustentador de su unicidad e irrepetibilidad, mientras que en otras ocasiones generalizan los comportamientos observados en situaciones diacrónica o espacialmente diferentes; los geógrafos llegan así, bien a los estudios regionales dando explicaciones particulares de la singularidad de cada región, o bien a la explicación de la fenomenología geográfica a partir de ciertos modelos de comportamiento que permiten a la Geografía una fácil ubicación en el marco de las ciencias empíricas. La etapa explicativa no sólo permite el desarrollo racional de "las geografías del futuro", sino que también posibilita la comparación entre factores geográficos confluyentes en un fenómeno en áreas distantes de la superficie de la Tierra, por lo cual ayuda a comprender la dinámica del fenómeno estudiado y facilita la toma de decisión para una actuación consciente sobre esa fenomenología.

La Regional y la Sistemática son las dos grandes tendencias de la etapa explicativa de la Geografía. Ambas desarrolladas como antagónicas: la primera como una afirmación de la originalidad de los saberes geográficos que,

${ }^{3}$ Los trabajos de $\mathrm{Ph}$. Buache se tornan explicativos alcanzando la superación de lo meramente descriptivo en la elaboración de mapas sobre el relieve submarino, cuando todavía las técnicas de investigación submarina no podian aportar un conocimiento cierto de lo que no podia observarse directamente. Su método de trabajo hubo de basarse, pues, en el principio de causalidad 
al tiempo, ahoga el avance de la Geografía como ciencia por la irrepetibilidad de sus situaciones; la segunda, como eficaz revulsivo de las posibilidades metodológicas y prácticas de los estudios geográficos, pero eclipsando la originalidad de la Geografía, que se ha ido dispersando en sus diversas ramas al tiempo que daba paso a nuevas parcelas de la ciencia conformando la Geodesia, la Geología, la Meteorología, la Oceanografía, etc. Precisamente de esta disposición a la compartimentación, nacen una serie de sucesivas crisis de identidad que han permitido a la Geografía su afirmación científica merced a diversos recorridos, a menudo descalificantes, que le han llevado a su actual estado en el que una indefinición de los contenidos permanece latente y sirve de alimento a una polémica que todavía no se ha cerrado. Parece que los estudios fisiográficos, que alcanzaron un mayor desarrollo, han gozado de más facilidades para desligarse de la tutela de la Geografía; ello se ha visto favorecido por la demanda que la sociedad ha hecho de sus respectivos campos de conocimiento, que han dado antes el paso de la ciencia teórica a la ciencia aplicada, es decir, de la fase explicativa a la etapa tecnológica (tal es el caso de la Geología, la Meteorología, o la Oceonografía, por poner algunos ejemplos), por lo que han sobrepasado la evolución de la rama de conocimientos que ha sido su matriz y se han definido con contenidos propios. Ante esa paradójica situación a que las tendencias sistemáticas han conducido a la Geografia, afirmándola en el marco de las ciencias modernas y despojándola de contenidos, los geógrafos han defendido su parcela refugiándose en las tendencias regionalistas, como señala Daus ${ }^{4}$ para quien los esquemas actuales "obligan a los geógrafos a adoptar el concepto regional como punto de partida de su estudio de la superficie terrestre". No obstante, el antagonismo conceptual entre lo regional y la sistematización ha servido para marginar el saber geográfico que oscila entre la explicación singular y la causal o, cuando menos, para cuestionar las posibilidades de avance de la Geografía, mediatizada por su propia originalidad. Cuando una disciplina no puede predecir comportamientos, aunque el tipo de predicción sólo sea probabilístico, esa disciplina deja de ser científica, porque no se apoya en las premisas que validan a un método como científico.

\section{El desarrollo tecnológico}

En sólo un siglo la Geografia ha alcanzado su "madurez" al pasar a ser una ciencia aplicada. Es cierto que como tal ciencia aplicada ha ofrecido

${ }^{4}$ Pág. 57 de Qué es la Geografia de OIKOS Asociación para la Promoción de los Estudios Territoriales y Ambientales. 6. ${ }^{a}$ edición. Mayo de 1982. Buenos Aires. 
sus servicios, ya desde el principio de siglo, e incluso prestó una estimable ayuda a la navegación y a los descubrimientos en la Edad Moderna, pero es a partir de la segunda mitad del actual siglo cuando es más común el hablar de Geografía Aplicada como ciencia con cultivadores decididos e institucionalizada en cátedras, departamentos universitarios y de investigación: la Geografía ha llegado a su etapa tecnológica. La tecnología, al tiempo que hace avanzar a las ciencias en su profundización teórica, genera y desarrolla métodos y técnicas propios encaminados a facilitar el uso de esos principios básicos de la teoría para tener de ellos la posibilidad de uso que mejor satisfaga las necesidades materiales demandadas por la sociedad. EI levantamiento y la interpretación cartográficas son unas de las principales demandas, aunque no las únicas, ya que el tratamiento y sintesis de grandes volumenes de información sobre los diferentes aspectos que actúan en estrecha interacción en la superficie terrestre, constituyen otra componente sustancial de la demanda global a la Geografía. Por todo ello, las ciencias geográficas necesitan apoyarse en el conocimiento de los comportamientos de los factores que actúan sobre la superficie de la Tierra, los resultados de las diferentes modalidades de acción, las modificaciones introducidas por las variaciones en los factores actuantes y los estados de máximo equilibrio natural, para que, a partir de tales premisas, pueda construir el modelo de intervención óptimo encaminado a obtener el máximo provecho social del entorno físico, sin deteriorar sensiblemente ese entorno hipotecándolo para el futuro. La planificación regional o el ordenamiento del espacio serian las consecuencias sociales de las posibilidades emanadas de la nueva etapa del saber geográfico, acatada por múltiples profesionales que comparten con el geógrafo esta nueva tarea, cuando no le desplazan definitivamente. Los estudios denominados de clasificación y usos de la tierra, iniciados hace ya varias décadas en el Reino Unido fueron los precursores de esta tecnologia geográfica que está llamada a investigar las condiciones físicas y antropogeográficas del espacio geográfico, la dinámica de sus tendencias y, en definitiva, las modificaciones que serían necesarias introducir en los factores que conforman unas condiciones definidas para favorecer, hasta el óptimo, la ocupación humana del suelo, o para mejorar el uso de la actual ocupación de cara a la intensificación del bienestar social. Para poder investigar en este sentido y presumir los resultados de las posibles acciones sobre el medio, se hace inescusable el conocimiento de las leyes que reglan la dinámica de los factores intervinientes en ese espacio y, por ello, la incontingencia de la metodologia sistemática para llegar a la tecnología. 


\section{Una visión sobre el camino recorrido por la Geografía Española}

Desgraciadamente no existe en España una institución consagrada a recopilar todo el cúmulo de documentos que, a lo largo de la historia, han sido publicados en nuestro país con temática y contenidos geográficos; esta práctica es común en Francia y en la mayor parte de países anglosajones, donde existen recopilaciones y sintesis anuales sobre la bibliografía geográfica aparecida ${ }^{5}$. A falta de ello, la evolución del pensamiento geográfico sólo se podrá hacer de forma parcial y, en el presente, habrá que mirar la organización académica de la Geografía para inferir desde ella las funciones actuales de la ciencia y, con ellas, la situación en la secuencia normal de la evolución y el progreso en materia geográfica.

La Geografia Española no es un caso atípico dentro de la evolución del pensamiento geográfico, aunque es cierto que, sobre todo en el siglo $x x$, ha progresado con un cierto desfase que le ha hecho ir a remolque de la evolución experimentada en Francia, y aún más atrasada con respecto al protagonismo alcanzado por las escuelas anglosajonas después de la Segunda Guerra Mundial. La fase descriptiva se mantuvo en España con el acervo productivo de los geógrafos musulmanes de Al Andalus, que imitando la cultura griega, resucitaron la geografía que narraba periplos, viajes exploratorios y exóticos, o que describía ciudades y caminos relacionándolos con su entorno ${ }^{6}$. Esta tradición permitió, durante la Edad Moderna, el agrupamiento de trabajos y autores en un sentir común de "escuela geográfica", con personalidad propia, que es mundialmente conocida como «escuela española", y cuyos trabajos no quedan sólo en el levantamiento de planos de los enclaves de población en las colonias (de los que se conservan valiosas muestras en el Archivo de Indias), sino que además comentan los itinerarios de los exploradores, transmitiendo la información de tales andanzas, por lo que paralelo a su valor geográfico corre su importancia histórica. Con algunos geógrafos españoles de la Edad Moderna, la Geografía se hace

${ }^{5}$ Tal es el caso del Research Catalogue de la American Geographical Society y del Current geographical publications para las publicaciones geográficas americanas, de Geo-abstract en el Reino Unido, de los Geographicsches Jahrbuch y de Documentatio Geographica para la bibliografía en alemán, de Referativnyi Zhurnal Geografiia para las rusas, o de Bibliographie geographique internationale para la literatura especializada francesa. Todas ellas por su extensión e importancia sobrepasan sus propias fronteras nacionales.

E El valor y la importancia de estas aportaciones les hace todavia sentirse orgullosos a los actuales geógrafos del ámbito de los paises musulmanes. Vease a Ahmad Nafis en «Some contributions to geography by Spanish Muslims" en la Pakistan G. Rev.; Lanore, 1954, t. IX, ก. 1. 
teoría y deja de ser descriptiva; asi ocurre con AGUIRRE (1782) que escribe una geografía física del Universo bajo el título de Indagaciones y reflexiones sobre la Geografía con algunas noticias previas indispensables ${ }^{7}$.

La etapa explicativa con plenitud de asentamiento es ya obra de los geógrafos del siglo xx y se desenvuelve dentro de la mimesis con la geografia francesa, por lo que participa tanto de sus virtudes como de sus defectos. La tendencia regional se erige en protagonista entre las metodologías y los intereses de los geógrafos españoles, siendo el modelo vidaliano el ejemplo seguido y repetido hasta la saciedad. Este colonialismo geográfico, tanto en lo conceptual como en lo metodológico y en la docencia, se debe fundamentalmente a que Francia institucionaliza la comunidad geográfica al menos 30 años antes que España, donde, a pesar de la antigüedad de la Real Sociedad Geográfica (cuyos miembros eran representantes de una variopinta mezcolanza de matemáticos, físicos, militares, nobles y exploradores), no comienza la profesionalización científica de la Geografia hasta después de la Guerra Civil. Toda esta carga actúa como una losa sobre el pensamiento geográfico reciente en España y, hasta finales de la década de los sesenta, la geografía española se mantiene al margen de las revoluciones conceptuales habidas en el mundo, permaneciendo fiel a los modelos excepcionalistas defendidos por la Geografía Regional; ello no es óbice para que algunos geógrafos sean llamados a colaborar en planificación en el marco de los Planes de Desarrollo que siguieron a la política de estabilización económica a partir de 1962, lo que plantea a estos profesionales una problemática inherente a la paradójica situación de estar utilizando la tecnologia de una disciplina que no había desarrollado las bases normativas de su propia ciencia. La ayuda prestada en tal situación se apoyaba más en el sentido común que en la ciencia geográfica y de ahí la falta de continuidad y de hueco que los geógrafos como colectivo encontraron con posteridad dentro de la comunidad técnica del país.

La normalización de las bases científicas de la Geografia es algo que comienza a sentirse en los departamentos universitarios en época tan tardía como es la de la segunda mitad de la década de los setenta, mientras que la falta de preparación metodológica y de dominio de las técnicas de tratamiento de la información y de la inferencia estadística, por parte de un buen número de profesores que aprendieron la Geografia como una materia me-

Recientemente reproducida en facsímil por Ediciones de la Universidad de Barcelona bajo la supervisión de Horacio Capel que tanto esfuerzo ha hecho por resucitar el pasado de la geografía hispánica. 
ramente descriptiva y explicativa sólo en sus aspectos particulares o singulares, han servido para dejar huérfana de padrinazgo a la corriente normativa. Esto justifica la falta de ímpetu en la irrupción de la nueva etapa, que queda en manos de los «jóvenes geógrafos", y explica la ausencia de publicaciones españolas, de contenido metodológico y conceptual, en las grandes reseñas bibliográficas de carácter internacional.

\section{VALOR ACTUAL DEL SABER GEOGRAFICO EN LA EDUCACION Y EN LA SOCIEDAD}

Hay geógrafos que se sienten pesimistas por el estadio permanente de crisis en el que se halla sumergida la Geografía, sin definirse plenamente, sin que vislumbre con claridad el tipo de concepto y método que se impondrán a los restantes entre las muchas ideas y tendencias que han aparecido en el período postbélico. En los últimos cuarenta años se produjeron más corrientes y contracorrientes geográficas que en los dos mil años anteriores, lo que, bien entendido, es mucho más positivo que la estabilidad derivada de una afirmación anquilosante sobre un concepto o una tendencia basada en la pseudoverdad de una ciencia definida en su totalidad. Las crisis son positivas, yo diria que hasta deseables ${ }^{8}$; sin ellas se oscurece el necesario espíritu crítico que sirve de impulsor creativo para el avance de las ciencias. Lo que no resulta tan positivo es el abandonismo en el que se sumen algunos cuando llegan esos "ríos revueltos" que son consustanciales a los periodos de crisis. El futuro de la Geografía lo estamos construyendo hoy mismo todos los geógrafos gracias a la insatisfacción que nace de los problemas epistemológicos presentes ${ }^{9}$, y ese futuro es, en definitiva, el resultado de las reflexiones intelectuales de este momento, pero también una consecuencia de la demanda de la sociedad del mañana que, en buena medida, será un reflejo de la educación que hoy proporcionamos a las generaciones que

a Ya me hice eco de esta postura en otro trabajo más especifico con motivo del homenaje a D. Antonio Domínguez Ortiz, que respondía al título de Filosofia y justificación de la actual crisis cientifica de la Geografia: la necesidad de las nuevas geografias y que fue editado por el Ministerio de Educación y Ciencia, 1980.

${ }^{9}$ Así lo refleja BERRY, B.J.L. en "Creating future geographies" A.A.A.G., 1980, 70, n. ${ }^{\circ}$ 4; y participan en esa idea todos aquellos que han entrado en un análisis profundo de las contradicciones y luchas existentes entre las diversas posturas mantenidas por los geógrafos contemporáneos. 
tendrán la responsabilidad de la decisión y de la gestión social en los tiempos venideros.

Llegados a este punto hay que comprometerse con la alta responsabilidad que recae sobre aquellos que nos encargamos de la educación de nuestros jóvenes generaciones, y no puede existir un compromiso responsable si, previamente, no hacemos un análisis crítico de aquello que enseñamos, de los contenidos y de los objetivos del saber geográfico. No hay mejor postura crítica que la que comienza por cuestionarse el valor del conocimiento de aquello que se erige en objeto de nuestra reflexión y, por ello, creo que en el inicio de este recorrido habrá que preguntarse por la necesidad de la Geografía en la sociedad actual, para ser coherentes con su inclusión en los programas educativos y con la preocupación didáctica de su enseñanza ${ }^{10}$. Son muchos los autores que estiman en la Geografía un alto valor educativo; algunos señalan incluso sus excelencias de manera pormenorizada:

"La realidad geográfica, debídamente expuesta y explicada incide en todas las facultades del espiritu y por ello es una disciplina de enseñanza de incomparable valor. La memoria y el sentido de localización y orientación, el raciocinio, en cuanto se explica en su coherencia - aunque sea elementalmente - el complejo espacial; la sensibilidad ante lo bello, cuando se representan ciertos cuadros de la geografía estética; la imaginación, cuando se evocan los países lejanos y sus formas de vida, son facultades intelectuales que la oportuna descripción geográfica despierta y estimula. Pero, por encima de todo, la geografía acicatea la facultad de la observación de las cosas concretas, el espíritu de método y orden, y la voluntad de conocer el mundo y el suelo nativo" ".

Sin entrar en un fervor como el que acabamos de señalar, creemos que la Geografía tiene un valor social que la justifica. Este valor social debe entenderse desde la propia sociedad y no por una necesidad egoísta de dar salida a cuantos integramos el grupo profesional de los geógrafos. Hasta el

10 Ya en otro trabajo nos hacíamos eco de esta preocupación y, de su reflexión, concluiamos en una postura muy próxima a la que ahora reflejamos. Me refiero a mi artículo "Hacia la búsqueda de una nueva concepción didáctica de la Geografia en el marco de las Ciencias Sociales" aparecido en el n. 248 de mayo-junio de 1983 de la Revista de Orientación Pedagógica BORDON, de la Sociedad Española de Pedagogía; págs. 245-270.

11 Cita de Federico A. DAUS en Qué es la Geografia, pág. 89 de la sexta edición de mayo de 1982. Editado por OIKOS Asociación para la promoción de los Estudios Territoriales y Ambientales. Buenos Aires. Argentina. 
momento presente, la Geografía no ha alcanzado el rango de ciencia aceptada y asumida, como lo han alcanzado aquellas que poseen tecnologías utilitaristas (Quimica, Física, Matemáticas, Medicina, Genética, etc.), por eso, su supervivencia hay que apoyarla en su utilidad, y ésta hay que documentarla convenientemente: ¿Para qué sirve la Geografía?

Si el espacio terrestre es el campo de actuación de los fenómenos geográficos, todos los aspectos que incidan en él, viéndose limitados en el mismo, modificándolo o perturbándolo, serán objeto del estudio geográfico; por tanto, la Geografia debe servir para dar a conocer los componentes del espacio físico y las leyes que rigen su equilibrada permanencia, para interpretar el espacio y el tiempo como dualidad vital en la sociedad de cualquier época y cultura, para investigar el desarrollo y el alcance de todos y cada uno de los procesos físicos que ocurren en la superficie de la Tierra; y, como consecuencia de esa triple función, informativa (ayuda al conocer), explicativa (favoreciendo la interpretación) e investigadora (contribuyendo a su desarrollo científico y a su uso tecnológico), la Geografía se abre un hueco imprescindible entre las disciplinas que deben contribuir a la educación del hombre, porque:

1) Le presta información referente a los organismos de respuesta de la naturaleza ante los usos que el hombre, como individuo o como colectivo, hace de ella;

2) le educa críticamente mediante la investigación de los resultados de las relaciones hombre-medio;

3) le concede instrumentos válidos para atender a modificaciones definidas en el espacio.

Si la Geografia informa, educa y puede acondicionar el medio transformándolo según una pauta deseada, debe ser considerada, desde el punto de vista social, como una ciencia útil, y su inclusión en los programas de enseñanza es una necesidad evidente.

Según los anteriores razonamientos, la aparente o auténtica inutilidad de la Geografía en la sociedad actual es sólo achacable a su inadecuada didáctica, o a su incorrecto uso, o al desconocimiento social de la misma. En cualquiera de los tres casos se hace ineludible una actuación de los geógrafos encaminada a efectuar un replanteamiento de los estudios geo- 
gráficos en todos los niveles y a desarrollar una campaña de prestigio de la Geografía como ciencia útil a través de una amplia difusión de sus posibilidades y usos.

\section{CONCIENCIA Y ANALISIS DE LAS DEFICIENCIAS DIDACTICAS}

Una ligera ojeada sobre los proyectos didácticos de la Geografía en los niveles elementales y medios nos muestra a una ciencia que se construye sobre unos supuestos regionales, que peca de extremadamente descriptiva y que adolece de una falta de base general tanto en la explicación de los fenómenos físicos de la superficie de la Tierra como en la tipificación de las relaciones hombre-medio y en el análisis de los resultados de esta relación. La enseñanza de la Geografía se efectúa de una manera muy pasiva y ello refuerza su desprestigio entre el alumnado; por otra parte existe como una dejadez o una falta de interés por investigar nuevas fórmulas didácticas y ello a pesar de que más del 90 por 100 de los geógrafos que trabajan su disciplina lo hacen como enseñantes. El número de trabajos geográficos que tratan sobre los temas didácticos no alcanza el 0,5 por 100 en ninguna de las siete grandes series de publicaciones anuales que recogen la bibliografía aparecida sobre temas geográficos ${ }^{12}$, lo que es especialmente grave, ya que, una materia que se enseña inadecuadamente compromete seriamente su rentabilidad futura y su permanencia social en el marco de la docencia. Y está claro que esta problemática puede generalizarse a todos los países del mundo, aunque hay algunos en los que se acentúa. España, por su dependencia externa en la investigación y en el desarrollo de la Geografía y por la estructura de la dedicación profesional de sus geógrafos (con mayoría aplastante como docentes), es uno de estos países en los que esta problemática alcanza un alto índice de gravedad.

Es cierto que, cada vez más, existe una preocupación didáctica por la geografia, pero las investigaciones se inclinan antes por la vertiente histórica,

${ }_{12} \mathrm{Ni}$ Geo Abstracts, ni Current geographical publications, ni New geographical literature and maps (revistas en lengua inglesa), ni Bibliographie Geographique Internationale (en lengua francesa), ni Geographicsches Jahrbuch o Documentatio Geographica (ambas en alemán), ni la rusa Referativny Zhurnal: Geografiia, que recogen prácticamente la totalidad de los trabajos de contenido geográfico y parageográfico que se publican anualmente en el mundo, recogen un 0,5 por 100 de temas dedicados a la Didáctica de la Geografía. 
analizando lo que se ha hecho, que por la pedagógica, buscando nuevas fórmulas ${ }^{13}$. Consecuencia de esa falta de iniciativa, del poco peso social del colectivo profesional de los geógrafos y de la inadecuada o miope visión del proyecto educativo actual de cara a la educación integral del hombre, es la situación actual de la Geografía en los programas de los planes de estudio: diluida, desplazada y postergada. Esta es una constante que se viene repitiendo en los últimos decenios y una permanente reivindicación de los geógrafos españoles desde que existen como tales. En 1957, el profesor Plans Sanz de Bremond, posiblemente el más preocupado de los geógrafos españoles por los temas de la didáctica, se cuestionaba la continuidad de la Geografía en razón de la irracionalidad e insuficiencia de la misma en la Enseñanza Media ${ }^{14}$, y en 1984 , algunos geógrafos de reconocido prestigio en el seno de la Real Sociedad Geográfica (RSG) y de la Asociación de Geógrafos Españoles (AGE), se siguen cuestionando la continuidad de la Geografía ante las perspectivas que se abren a esta disciplina con vistas a la inminente reforma de las Enseñanzas Medias ${ }^{15}$. Esta precariedad y falta de atractivo de la Geografía es, en buena medida, una consecuencia directa de sus deficiencias didácticas, y un buen avance de la investigación didáctica no puede hacerse sin partir de un análisis de las deficiencias actuales.

Es lógico pensar que las necesidades didácticas de una ciencia están en consonancia con la finalidad y contenidos de la misma y con las carac-

${ }^{13}$ Incluso los grandes investigadores en la materia, como N.J. GRAVES, se pierden frecuentemente en esas narrativas; véase "Geographical education" en Prog. hum. geogr., 1981, $5, n .{ }^{\circ} 4(\mathrm{~GB})$, en cuyo artículo, el autor citado, filosofa sobre el lugar de la epistemologia y la hisiuria de la Geografía, describiendo las estrategias de la enseñanza de la Geografia en varios paises, pero sin hacer la posible critica a las mismas, ni dar las alternativas más útiles a tales estrategias.

${ }_{14}$ En un articulo aparecido en el Correo Español - El Pueblo Vasco de Bilbao, en 1957, y que es reproducido en Orientaciones sobre la didáctica de la geografia, libro editado por Magisterio Español bajo el patrocinio del Ministerio de Educación, en 1970 (págs. 51-54), bajo el titulo de "¿Ha desaparecido la Geografía del Bachillerato?». Pedro PLANS se hacia eco de unas palabras del profesor Casas Torres en las que decia: "La geografía sigue siendo en España una ciencia desconocida, quizá porque nuestros planes oficiales de Bachillerato se encargan de que millones de españoles la odien desde los once años y la ignoren desde los trece"s.

${ }^{15}$ En cada reunión de la AGE (Asociación de Geógrafos Españoles) se crea una comisión encargada de analizar la situación de la Geografía en los planes de enseñanza (EGB y Bachillerato), cuyos trabajos nunca tienen un reflejo positivo y terminan en el olvido. Ante la nueva reforma de las Enseñanzas Medias. Adela Gil y José Estébañez, entre los geógrafos de la permanente de la RSG (Real Sociedad Geográfica), se proponen elaborar una alternativa al plan que se intenta implantar, ya que, se siente una especie de rubor e indignación entre los geógrafos por la posición y contenidos que le son asignados a los estudios de geografía en los nuevos programas. 
terísticas del educando que la estudia; consecuentemente el proyecto didáctico será diferente en la Enseñanza General Básica, en los Estudios Medios y en los Estudios Universitarios. A las características didácticas generales de la enseñanza de la Geografía habrá que sumar las especificas de cada ciclo educativo y con ello construiremos el cuerpo teórico que define la Didáctica de la Geografía.

¿Cómo se enseña la Geografía en el momento actual?: En términos generales, se enseña como una "ciencia de aula" (con pocas salidas al medio natural o humanizado), que estudia espacios concretos (abuso de la Geografia Regional y de la metodología descriptiva), que se auxilia del Atlas como instrumento de trabajo con finalidad más corográfica que explicativa (generalización de la descripción e incluso de la enumeración toponimica) y que posee como particularidad metodológica más acusada el ser «una ciencia de síntesis" (por lo que olvida frecuentemente el análisis de las componentes que forman parte del fenómeno geográfico perdiendo la posibilidad de inferir o de deducir pautas de comportamiento simples y normalizadas dentro de unos esquemas). De esta situación, que es un reflejo de "la petición de parte" que suponen los planes de estudio actuales, se derivan las principales deficiencias de la Didáctica General de la Geografía:

1. Exceso de pasividad que ha sido frecuentemente denunciado por muchos docentes pero que tiene una dificil solución. La pasividad es una tónica de la enseñanza en España. La incoherencia entre el objeto de la Geografía, que es el estudio del espacio, y su didáctica como ciencia que se enseña preferentemente en el aula, es una componente más que contribuye a la pasividad. Una enseñanza fundamentalmente descriptiva, con fuerte componente memorista, dificulta la docencia participativa e interactiva profesor-alumno.

2. Abuso del estudio de lo concreto como finalidad, en una especie de ensimismamiento particularista (Geografia de España, Geografía de Andalucía, el Río Guadalquivir, la Penibética, etc., son títulos normales en estudios geográficos), olvidando la observación de la fenomenologia geográfica (los relieves, los climas, las redes hidrográficas, el poblamiento, etc.) y la tipificación de tales observaciones, para deducir de ellas generalizaciones suceptibles de ser aplicadas a la explicación de los fenómenos concretos en una región o espacio determinado.

3. Falta de instrumentalización, ya que no se les suelen enseñar a los alumnos las técnicas que han permitido pasar del espacio real a su represen- 
tación gráfica en los mapas; por ello, los atlas no son utilizados en todas sus posibilidades; a veces no se entiende una relación clara entre el medio geográfico y lo representado en el mapa, siendo para el alumno un instrumento con un lenguaje criptográfico que no acierta a interpretar. Sería necesario que se potenciara la observación, como método, con la práctica de la representación realizada por el alumno para expresar sus propias observaciones. Al mismo tiempo, y en la medida en que se asciende en los estudios geográficos, se hace imprescindible el manejo de otros instrumentos de análisis como la fotointerpretación, la informática o la encuesta social.

4. Insuficiente relación entre saber geográfico y las necesidades de la vida cotidiana, o lo que es igual, abuso del conocimiento teórico, en un afán de erudición desconectado del mundo real del entorno físico del alumno. Es un ejemplo de ello el alumno de la costa mediterránea española que desconoce las causas de las inundaciones que periódicamente sufren sus tierras y, sin embargo, estudia y se aprende el mecanismo de los monzones. Lo normal es que se utilizaran los conocimientos básicos de la Geografía General para explicar los comportamientos que se observan en el medio próximo; de esta forma se puede efectuar esa racional integración hombremedio que es necesaria para la correcta utilización del patrimonio geográfico en provecho de todos y respetando los derechos de las generaciones futuras.

Estas deficiencias se ven potenciadas aún más por la falta de acomodación didáctica al alumno al que se dirigen los programas. Son escasas, y a veces nulas, las salidas a la calle y al campo de los cursos que se inician en el estudio de la Geografía, lo que limita enormemente las posibilidades de aprendizaje, ya que, un alumno de 8, 9 ó 10 años (edades de los discentes del actual ciclo medio de la EGB en España en el que aparece por primera vez la Geografía en los programas educativos) no está preparado para captar y comprender el espacio en su forma abstracta y, por tanto, tendrá dificultades para estudiar la fenomenología geográfica. Esta inicial dificultad, unida a cualquiera de las deficiencias generales que hemos enunciado anteriormente, están en la base de posteriores posturas de bloqueo o de rechazo a la geografia. Lo triste es que esas inadecuaciones didácticas son conocidas por los geógrafos desde hace mucho tiempo, como ya señalaba Plans en $1962{ }^{16}$, continúan en

${ }^{16}$ Véase el artículo publicado en Vida Escolar, págs. 39-42 del n. ${ }^{\circ} 35-36$, revista del Centro de Documentación y Orientación Didáctica de la Dirección General de Enseñanza Primaria (Madrid); en aquel $n .{ }^{\circ}$ de enero-febrero de 1962 y bajo el titulo de "La Geografia Humana en la Escuela", el profesor PLANS insistía en la necesidad de una enseñanza activa, con un método de trabajo basado en la salida habitual del aula a la calle. Este articulo ha estado luego reproducido en el mismo libro que referenciábamos en la nota 14. 
la actualidad y habiendo sido modificados sólo parcialmente los defectos ya denunciados en aquella época. Es labor del geógrafo hacer ver a los responsables del Ministerio de Educación y a los docentes, la necesidad de salvar esas inadecuaciones didácticas; y desde que el geógrafo tiene su asociación profesional que, a nivel institucional, representa las aspiraciones de todo el colectivo (por lo que tendrá una mayor audiencia pública que el individuo aislado), es a ésta a quien corresponde esta función concienzadora ${ }^{17}$.

Las modificaciones didácticas y curriculares introducidas por los Programas Renovados del Ministerio de Educación y Ciencia para los ciclos inicial y medio de la EGB, están más en línea con una pedagogía activa y con la utilización de fórmulas didácticas más próximas a las que aquí estamos pidiendo ${ }^{18}$. Sin embargo, la desconexión se vuelve a plantear en el Bachillerato al que los alumnos llegan a la edad de 14 años (15 años en su primer curso de Geografía ubicado en el segundo de BUP), cuando ya han madurado en las capacidades de abstracción y sintesis, por lo que pueden dominar la deducción y pueden preveer interrelaciones. Es el momento en el que nuestro educando debe desarrollar su sentido crítico y sus posibilidades investigadoras en Geografía, para lo cual necesitaria avanzar sustancialmente en las metodologias que le permitirán dar ese salto cualitativo: tratamiento de datos, técnicas de muestreo, inferencia estadística, tipificación de espacios, etc.; sin embargo, el estancamiento es manifiesto. Una Geografía Económica del Mundo que no conecta con la necesaria crítica a la sobreexplotación de los espacios o de los pueblos, que no se proyecta en una instrumentalización de técnicas para analizar otras realidades diferentes a las contenidas en el programa, que no se estructura sobre el trabajo de la experiencia inmediata (lo que ocurre en su entorno geoeconómico), etcétera. Ahi se vuelve a convertir la Geografía en una ciencia informativa, sin utilidad social inmediata, por lo que tendrá menos atractivo del que podría tener para el alumno.

En la enseñanza superior predomina una didáctica basada en la lección magistral, aunque los recursos didácticos son bastante más amplios, pues, la especialización y la variedad de materias, y con programas no bien definidos, permiten al profesor preparado hacer tan atractiva su clase como

17 Nos referimos a la AGE, que junto a la Real Sociedad Geográfica, debian trabajar en España por racionalizar los proyectos de estudio en sus contenidos geográficos y por prestigiar a la Geografía en el ámbito social.

${ }_{18}$ Ello lo suscribiamos detalladamente en las pags. 247-254 del artículo anteriormente citado en la llamada 10. 
imaginación sea capaz de derrochar. En la enseñanza superior la madurez del educando es total y, por tanto, no hay limitaciones en cuanto a las posibles fórmulas didácticas: inducción, deducción, análisis, síntesis, observación, abstracción, simplificación de la realidad espacial mediante el establecimiento de modelos matemáticos, etc.; todo puede ser utilizado y, de hecho, se utiliza, aunque no exista norma alguna que permita generalizar la existencia de una didáctica desarrollada.

\section{POSIBILIDAD DE RACIONALIZAR LOS ESTUDIOS DE GEOGRAFIA EN LOS PROGRAMAS EDUCATIVOS}

Si admitimos que la Geografía tiene un determinado valor social y, por ello, un hueco dentro de los proyectos educativos; si observamos que no cumple con su función educadora y presumimos que esa incompetencia es un producto de la inadecuada forma de enseñarla; si, como geógrafos, estamos convencidos de que existen alternativas más útiles desde el punto de vista didáctico, lo lógico es que hagamos un esfuerzo por mostrar esas otras fórmulas a través de un proyecto metodológico y curricular para cada uno de los niveles educativos: ¿Cómo pueden racionalizarse los estudios de la Geografia? La respuesta más adecuada a tal pregunta sería la de dar a cada nivel los conocimientos que precise con el método más atractivo y eficaz; eso es lo que pretendemos de una forma breve sin mayores ambiciones.

En términos generales podemos considerar tres tipos de niveles educativos esenciales: el básico, el medio y el superior.

1. El básico es el que corresponde a cualquier individuo de la sociedad, que podrá continuar estudios o terminar con la finalización de este ciclo, $y$, por cuya razón, en él deberá adquirir todos los elementos geográficos básicos que consideremos imprescindibles para el ciudadano normal.

2. El nivel medio puede ser un paso intermedio para el nivel superior, o tener una razón en sí mismo dando como resultado la salida del individuo a la vida profesional dentro de la sociedad.

3. El nivel superior tendrá siempre un carácter de especialización y el estudiante de este nivel debe dominar los instrumentos del saber geográfico 
que luego tendrá que utilizar, ya sea como función básica de su profesión (para el geógrafo), ya como función complementaria y anexa a su profesión (para el geólogo, el economista, el sociólogo, o para determinados ingenieros y profesionales de la técnica).

Los dos primeros niveles deben contemplar prácticamente la misma información aunque con diversa profundidad. En el nivel superior hay que tener presente la especificidad de la especialidad de los alumnos a quienes va dirigida la Geografia.

\section{La Geografía en la EGB}

En este nivel, la enseñanza de la Geografía debe preparar al alumno para entender el contexto físico en el que se desarrollan sus propias experiencias sociales, debe formarle una conciencia capaz de valorar y respetar el patrimonio natural del espacio que habita, debe permitirle relacionar la información geográfica con la información política y social que diariamente le llega a través de los medios de comunicación de masas.

La didáctica debe responder a las exigencias de una metodología que permita al alumno una participación máxima en su proceso de formación. La tan cacareada enseñanza activa no siempre es fácilmente reproducible en la práctica didáctica, ya que es necesario conocer la evolución psicológica de los alumnos y motivarlos (en función de esa evolución) con la garra suficiente como para despertar su interés, pero tampoco es un problema de difícil solución ${ }^{19}$.

El alumno de hasta 10 años (el de los ciclos inicial y medio de la EGB) tiene una enorme curiosidad intelectual que debe orientársele hacia la búsqueda de objetos (primero) y hechos (más tarde) que puedan ser situados en el espacio en relación con el propio alumno, lo que le permitirá adquirir de forma intuitiva la noción de ese espacio. La observación será el método de trabajo básico con los alumnos de estas edades; la salida del aula (al

19 La participación activa del alumno en clase no tiene por qué ir ligada con una concepción moderna de lo geográfico. ÁLVAREZ MÁRQUEZ en La Geografía Activa en la Escuela, de Editorial Escuela Española S. A. (Madrid 1983), derrochando imaginación y voluntad, muestra cómo desde un planteamiento muy tradicional de la enseñanza puede llegarse a una dinámica de perfecta participación. 
propio colegio o a la calle) será una práctica muy útil. La actividad se desarrollará en torno a la propia experiencia del alumno que deberá reproducir sus observaciones espaciales a través de croquis o esquemas, con lo que alcanzará las nociones de plano y mapa. En esas representaciones habrá de diferenciar entre espacios organizados por el hombre (la calle, el barrio, el pueblo o la ciudad, los campos de cultivo, los caminos y vías de circulación, etc.) y aquellos que no lo están. Deberá centrar su atención sobre fenómenos y hechos naturales que se dan en el espacio (como el relieve, las liuvias, el calor o el frío, etc.), aprenderá a representarlos en abstracto mediante el dibujo y luego deberá pasarlos al plano. Será necesario que se familiarice con las unidades de medida espaciales (el $\mathrm{m}^{2}$, la $\mathrm{Ha}$., el $\mathrm{Km}^{2}$ ), con las formas espatiales, con los instrumentos de medición fisica (el altímetro para el relieve, el pluviómetro, el termómetro) y con las técnicas para detectar las preferencias de uso (la entrevista o la encuesta). Por último, habrá que enseñarle a organizar y a disponer su información para su posterior uso.

Con el alumno de 11 a 14 años (ciclo superior de la EGB) hay que desarrollar la técnica de la inferencia de los comportamientos geográficos a partir de las observaciones. Hay que analizar esos comportamientos en su entorno y en áreas más alejadas. El trabajo con el atlas (instrumento de representación de las relaciones en el espacio) se hace imprescindible, y la extrapolación, por analogía o por divergencia, de las propias experiencias a otras áreas será un buen ejercicio que deberá contar con el apoyo técnico de la diapositiva, de la fotografia, del libro y de los mapas y atlas. Para evitar que se introduzcan posturas de recepción más o menos pasivas (el alumno espera que el profesor o el libro le informen, mientras que él acepta y memoriza), se potencirá la interpretación de los hechos del espacio observable cirectamente y del representado en las diapositivas y en los textos. La práctica y el ejercicio sobre el mapa, el atlas, la fotografía aérea o la fotografía de satélite, deberán introducir al alumno en la valoración de las distancias, y la introducción de los transportes como fórmula de explotación del espacio desarrollada por las sociedades, servirá para introducir la idea de los espacios relativos en función del tiempo que se tardará en recorrerlo o del costo del recorrido. Superficies y distancia son dos componentes espaciales cuya valoración habrá que efectuar mediante la realización de ejercicios que contemplen como unidad de comparación o como punto de referencia al espacio vivido por el alumno (su propio municipio, su provincia, su región o España); de tal forma que, a la región habrá que ir partiendo desde la provincia, a España desde la región, al resto de las áreas socioeconómicas desde España. La ampliación de la instrumentación utilizada en las observaciones geográficas deberá ir acompañada de la organización y tipificación de las informaciones; ello permitirá diferenciar climas, funciones urbanas, 
desarrollo social, etc. La excursión geográfica, los campamentos de 3 o 4 dias, la proyección de películas o vídeos (que debieran ser desarrollados por las instituciones educativas del país) y la realización de encuestas sociogeográficas, son procedimientos adecuados para una metodología atractiva y plena de actualidad.

El alumno que ha terminado la EGB estará en condiciones de entender, las predicciones del tiempo, las posibilidades económicas de un emplazamiento geográfico, los riesgos de la desforestación y de la pérdida de potencialidad del medio natural; sabria distinguir las razones geopolíticas de un conflicto 0 de una alianza; comprendería y podría juzgar los planes de ordenamiento del espacio, lo que le permitiría una mayor influencia en la gestión democrática del medio. En definitiva, sería un ciudadano más potenciado para una sociedad más participativa y se sentiría más capaz en el juzgar, en el opinar y en el actuar.

\section{La Geografía en las Enseñanzas Medias}

Los alumnos de este nivel (entre los 15 y los 18 años) están en la fase final de su desarrollo intelectual y están en condiciones de abstraer de la información concreta las leyes que regulan la dinámica de los hechos. A tal capacidad habrá que darle contenido geográfico mediante la participación del alumno en la elaboración de modelos de comportamiento geográficosocial que se plasman en las formas de vida (del hombre de la montaña, del hombre del valle, del hombre de la costa, o del hombre del desierto), o en las posibilidades de ocupación del espacio (áreas agrícolas, recreativas, industriales, comerciales, etc.). Deberá saber extrapolar los comportamientos o las leyes observadas en un área a otros espacios donde la confluencia de factores tenga una disposición parecida. Es necesario que profundice en los mismos contenidos que ya fueron objeto de su estudio cuando estudiaba la EGB; es un nuevo ciclo que tiene como base los mismos contenidos.

La metodología puede ser potenciada con el uso de la informática para el manejo de grandes masas de información (para su archivo y para acceder a ellas cuando se necesite efectuar una consulta) ${ }^{20}$; además deben intro-

${ }^{20}$ Esta es una posibilidad contemplada por algunos centros o profesores. Véase a T. HATT, en "Géographie et ordinateur au Lycée", en la Rev. geogr. Est. FR. 18, n. ${ }^{\circ}$ 3, año 1978; o a J. P. COLE y P. M. MATHER, en "The use of minicomputers in Geographie teaching.-Some prognostications", Geoforum, G.B., 1979, 10, n. ${ }^{\circ} 3$.

Todos ellos destacan el papel que pueden desempenar la informática en la futura enseñanza de la Geografía. 
ducirse las técnicas de la fotointerpretación. Permanecerán en uso todos los recursos didácticos desarrollados en el nivel anterior.

La racionalización del plan educativo debe pasar por la concreción de un determinado número de proyectos de trabajo que valgan para comprender la mecánica de las relaciones geográficas en toda su intensidad. Los proyectos de trabajo deben estar relacionados con experiencias, demandas - situaciones sociales que pueden sentar la base de algunos centros de interés como, la evaluación del potencial biológico de un espacio natural y el estudio de las técnicas y fórmulas que permitan su conservación, el estudio de la habitabilidad de los barrios y ciudades, la construcción de planos locales donde se definan los usos preferenciales del suelo, la elaboración de mapas temáticos regionales, de España o de espacios geoeconómicos supranacionales, etc. La relación y el establecimiento de conexiones o semejanzas del proyecto estudiado con la dinámica geográfica en otros espacios debe ser un descubrimiento que debe realizarse por los alumnos a través de las informaciones proporcionadas por la estadística y ofrecidas por el texto o por el profesor. Observando, tipificando las observaciones, construyendo modelos y relacionando fenómenos diferentes en el mismo espacio, o iguales en espacios distintos, el alumno de Media irá profundizando en su saber geográfico y construyendo su propia geografía.

Los proyectos de trabajo deben incluir también los temas que interesen a los alumnos y que sean propuestos por ellos; al tiempo, hay que contemplar que el tratamiento dado a cada proyecto debe gratificar las fórmulas nuevas y la creatividad en la investigación.

El alumno que ha terminado el nivel medio de estudios, debe comprender e interpretar críticamente las acciones de los gobernantes, las suyas propias y las de los demás, sobre el medio ambiente; debe saber localizar e interrelacionar las grandes áreas geoeconómicas y geopolíticas, conocer sus bases geofisicas y el mecanismo que las rige; debe leer correctamente todo tipo de mapas temáticos y topográficos, debe dominar la técnica de la representación esquemática de fotografias de paisajes y formas geográficas $y$, consecuentemente, debe saber interpretar tales gráficos. Debe haber adquirido una conciencia responsable como gestor de una parcela espacial del medio que le rodea y como educador de sus vecinos menos formados.

\section{La Geografia en los niveles superiores}

En la enseñanza universitaria no existen restricciones didácticas en razón del alumno; sin embargo, será necesario contemplar una racionali- 
zación en la oferta de los contenidos y métodos de tipo geográfico que deben ser objeto de estudio para aquellos que hayan elegido la Geografia como opción en sus estudios superiores, o para aquellos que la necesitan como complemento a sus estudios especificos.

El proyecto geográfico para los estudios universitarios debe incluir tres aspectos de la ciencia geográfica: el aspecto de la prospección, es decir, el que corresponde a la investigación; el aspecto didáctico, o aquel que está ligado con la enseñanza; el aspecto técnico, o el que supone la aplicación práctica de la Geografía ${ }^{21}$.

En la especialidad donde la Geografía sea sólo una materia complementaria, debe primar el aspecto técnico: geografías aplicadas según su dimensión económica, política, biológica, fisica, etc. La Geografía como especialidad en las Facultades de Geografía e Historia o Filosofía y Letras, debiera tener siempre, al menos, las tres ramas siguientes: Rama de investigación, rama de docencia, rama tecnológica.

Todos los principios didácticos señalados en los niveles básicos y medios son de vigencia en el nivel universitario, mientras que la especialización exigirá una profundización en ciertas técnicas como la informática, la fotointerpretación, la cartografía, la estadística, o la utilización del gabinete experimental, del vídeo y de la proyección cinematográfica, como auxiliares materiales.

${ }^{21}$ Sin definición aparente, estas posibilidades constituyen ciertas fórmulas de especialización en algunas universidades de Bélgica, Estados Unidos. Hungria, Reino Unido, Rumanía, Suecia y Unión Soviética. 


\section{BIBLIOGRAFIA}

Bibliografía que puede ampliar algunos de los aspectos teóricos desarrollados en este trabajo.

BARTON, B.: «Geographical inquiry in a hypothetical world». Prof. Geogr. 1978, 30, n. ${ }^{\circ}$ 4. USA.

BIDDLE, D. S.: "Developing conceptual understanding in Geography». Philip. geogr. J. 1978, 22, n. ${ }^{\circ} 3$. Filipinas.

CELANT, A.: «Riflessioni in tema di didactica della geografia». Ann. Pub. Istr. 1977, 23, n. ${ }^{\circ}$ 6. Italia.

COle, J. P.; MATHER, P. M.: «The use of minicomputers in geographie teaching. Some prognostications». Geoforum, 1979, 10, n. ${ }^{\circ}$ 3. Gran Bretaña.

DENNISON, V. D.: «The use of geography». Geography, 1981, 66, n. ${ }^{\circ}$ 4. Gran Bretaña.

FICK, K. E.: «Schulgeographie heute. Fachliche, didaktische, unterrichtspraktische Beiträge" (La geografia en la escuela hoy. Contribuciones técnicas, didácticas y prácticas). Frankfurter Beiträge zur Didaktik der Geographie, 2, 1978. RFA.

FOSTER, L. T.; JONES, K. G.: “Applied geography: an educational alternative». Prof. Geogr. 1977, 29, n. ${ }^{\circ}$ 3. USA.

Graves, N. J.: Geography in education. Heinemann. London, 1975. $\mathrm{VIII}+232$ págs.

-: «Geographical education». Prog. hum. Geogr., 1981, 5, n. ${ }^{\circ} 4$, G. B. 
Graves, N. J.: “International aspets of geographical education". Journal of Geography. $80, n{ }^{\circ} 3,1981$. USA.

HATT, T.: "Informatique, statistique et géographie quantitative au Lycée». Inf. géogr. 1977, 41, n. ${ }^{\circ}$ 3. Francia.

HENRIET, J. M.: "Les tendances nouvelles dans l'enseignement de la géographie». Esp. géogr. 1977, 6, n. ${ }^{\circ} 4$. Francia.

HiLL, A. D.: «A survey of the global understanding of American college students: a raport to geographers". Prof. Geogr. 1981, 33, n. ${ }^{\circ}$ 2. USA.

JACKSON, J. N.; FORRESTIER, J.: Practical geography: strategies for study. W. Heffner \& Sons. Cambridge, 1975. 320 págs.

JANDER, L.: Wissenschaft und Didaktik der Geographie. Eine Untersuchung zur Legitimation und Konstruktion geographischer Lerninhalte (Ciencia y didáctica de la Geografía. Investigación para legitimar y construir el contenido de la enseñanza de la Geografía). Gesamthochschule Kassel, 1976. 311 págs.

PLANS, P.: "Algunos aspectos de la enseñanza de la Geografía en la Universidad" Rev. Geogr. Barcelona, 1975. 9, n. ${ }^{\text {os } 1-2 .}$

-: «Los principios fundamentales de la metodología geográfica aplicados a la enseñanza con alumnos de EGB y Bachillerato". Did. Geogr. 1977. n. ${ }^{\circ}$ 2. Murcia.

--:Orientaciones sobre la didáctica de la Geografía. Magisterio Español. 1970. Madrid. 2. a edición. 183 págs.

Rey Balmaceda, R. C.: «Apreciaciones acerca de la formación del geógrafo y del profesor de Geografía". Bol. Soc. Argent. Est. Geogr. "Gaea». 1980. N. ${ }^{\circ}$ 99. Argentina.

SCHRAND, H.: «Neuorientierung in der Geographiedidaktik? Zur Diskussion um geographiedidaktische Strukturgitter» (¿Nuevas orientaciones de la didáctica geográfica? Discusión sobre su estructura). Geogr. Rundsch. 1978, 30, n. ${ }^{\circ}$ 9. RFA. 
SPERLING, W.: "Über die Ausbildung der Geographielehrer in der Deutschen Demokratischen Republik» (Formación de los profesores de Geografía en la RDA). Geogr. Z. RFA (1976). 64, n. ${ }^{\circ} 2$.

THEURILLAT, P. Y.: «Pour un renouvellement de l'enseignement de la géographie: la répartition des commerces dans une ville, la Chaux-de-fonds (canton de Neuchâtel)". Bull. Soc. Neuch. Géogr. 1980, n. ${ }^{\circ}$ 25. Suiza.

THOMAS, S. M.: "Some notes on the status and nature of field method courses at colleges and universities in the United States and Canada». Prof. Geogr. 1978, 30, n. ${ }^{\circ} 4$. USA.

VICKERS, A. F.: Patterns of interaction. Longman Cheshire, 1977, Melbourne. 123 págs.

VOLF, M. B. y otros: Geography education in the USSR and ways of improving it. Sov. Geo. 1976, 17, n. ${ }^{\circ}$ 9. USA.

WALFORD, R.: Geographical education in Britain. Prog. hum. Geogr. 1977, 1, n. ${ }^{\circ}$ 3. Gran Bretaña.

WILLIANS, M. (ed): Geography: a report of a working group, set up by the Education Standing Committee of the Geographical Association. London. Ward Lock Educational. Language teaching \& learning. Voll 2. 1981.76 págs.

YERMAKOV, Y. G.: "Some observations on geographie education in American universities" Sov. Geogr. 1978, n. ${ }^{\circ}$ 3. USA. 